\title{
miR-429 inhibits migration and invasion of breast cancer cells in vitro
}

\author{
ZHI-BIN YE ${ }^{1}$, GANG MA $^{2}$, YA-HUI ZHAO ${ }^{2}$, YUN XIAO $^{3}$, YUN ZHAN $^{2}$, \\ $\mathrm{CHAO} \mathrm{JNG}^{2}, \mathrm{KAI} \mathrm{GAO}^{4}$, ZHI-HUA LIU ${ }^{2}$ and SHENG-JI YU ${ }^{1}$ \\ ${ }^{1}$ Department of Orthopedics and ${ }^{2}$ The State Key Laboratory of Molecular Oncology, Cancer Hospital (Institute), \\ Chinese Academy of Medical Sciences and Peking Union Medical College, Beijing 100021; ${ }^{3}$ College of Bioinformatics \\ Science and Technology, Harbin Medical University, Harbin, Heilongjiang 150081; ${ }^{4}$ Institute of Laboratory Animal Sciences, \\ Chinese Academy of Medical Sciences and Peking Union Medical College, Beijing 100021, P.R. China
}

Received September 10, 2014; Accepted October 23, 2014

DOI: $10.3892 /$ ijo.2014.2759

\begin{abstract}
Accumulating evidence indicates that microRNAs (miRNAs) are involved in regulating cancer invasion and metastasis, and an increasing number of research demonstrates that miRNAs can promote or inhibit cell motility depending on genetic background of different cancers and the microenvironment. In the present study, we established an in vivo bone metastasis model of breast cancer by injecting MDA-MB-231 cells into the left ventricle of nude mice, and then screened the differentially expressed miRNAs between parental and bone-metastatic MDA-MB-231 cells using miRNA array. The results revealed that decreased expression of miR-429 was probably involved in negatively regulating bone metastasis of breast cancer cells. On the other hand, overexpression of miR-429 in MDA-MB-231 cells remarkably suppressed invasion in vitro. We identified ZEB1 and CRKL as potential targets of miR-429 by analyzing combined results from in silico search and global expression array of the same RNA samples. Immunoblot assay confirmed that miR-429 reduced their expression at protein level. Taken together, our results offer an opportunity for further understanding of the recondite mechanisms underlying the bone metastasis of breast cancer.
\end{abstract}

\section{Introduction}

Skeleton is the most common site of metastasis in breast cancer, which occurs in over $70 \%$ of patients suffering this disease. Bone metastasis, which seriously deteriorates the patients' life quality, is life-threatening and leads to several complications or skeletal-related events, such as pathologic

Correspondence to: Dr Sheng-Ji Yu, Department of Orthopedics, Cancer Hospital (Institute), Chinese Academy of Medical Science and Peking Union Medical College, Beijing 100021, P.R. China E-mail: shengjiyu@126.com

Key words: miR-429, ZEB1, CRKL, breast cancer bone metastasis fracture, pain, disability and nerve compression (1). Although huge efforts have been made in breast cancer research, what we can do is still limited with respect to bone metastasis of breast cancer. Safe and effective treatment to prevent or treat bone metastasis require sufficient validated targets. Hence, further insights into the molecular mechanisms underlying the bone metastasis of breast cancer are urgently needed (2).

The spreading from primary site to distant bones of breast cancer cells is very complex involving invasion, intravasation and extravasation of capillary vascular system, and locating at as well as flourishing in bones. Especially, various factors that promote cancer cell migration and invasion play a vital role in the process. It is widely accepted that tumor cells could travel to distant organs because of some acquired genetic alterations that facilitate invasion and metastasis (3).

Recent investigations in the field of cancer cell metastasis rely heavily on the availability of in vivo animal models. An ideal animal model should mimic the process of breast cancer cells developing spontaneously metastasis in distant organs. However, the animal models of bone metastasis we can build just represent unique stages of human breast cancer bone metastasis, for this phenomenon occurs rarely in animals $(4,5)$.

miRNA is a class of endogenous small non-coding RNAs which could modulate gene expression. In almost all cases, these RNAs negatively regulate gene expression via RNA-RNA binding to the $3^{\prime}$ untranslated region of target mRNAs in a manner of imperfect complementary, which then leads to either mRNA degradation or translational inhibition. Accumulating evidence demonstrates that miRNAs play crucial roles in tumor initiation and progression, which is exemplified by the ability to regulate every aspect of tumor pathological process including proliferation, migration, invasion, differentiation and escape from senescence and apoptosis (6-9). For example, aberrant expression of miR-106b promotes hepatocellular carcinoma cell migration in vitro and metastasis in vivo by stimulating epithelial-mesenchymal transition (10). miR-145, on the other hand, inhibits the migration and invasion of metastatic melanoma cells (11).

Several miRNAs have been found to be involved into breast cancer metastasis. For instance, miR-720 suppresses invasion and migration in breast cancer cells by targeting 
TWIST1 (12). miR-29b regulates migration of human breast cancer cells by modulating PTEN expression (13). In the present study, we established a bone metastasis mouse model by injecting MDA-MB-231 cells into the left ventricle and then compared the differentially expressed miRNAs between the parental malignant cells and the metastatic cells derived from the bones. The distinct expression pattern of the miRNAs provides important insight into the molecular mechanism of bone metastasis of breast cancer. Especially, we focus on the biological function of miR-429 through its target genes ZEB1 and CRKL.

\section{Materials and methods}

Cell culture and animals. Human mammary carcinoma cell line MDA-MB-231 was purchased from the American Type Culture Collection (ATCC, Manassas, VA, USA) and the cells cultured in the Leibovitz L-15 medium supplemented with $10 \%$ fetal bovine serum (FBS), streptomycin $(100 \mathrm{mg} / \mathrm{ml})$ and penicillin $(100 \mathrm{U} / \mathrm{ml})$. The same culture medium, adding $200 \mu \mathrm{g} / \mathrm{ml} \mathrm{G} 418$ (Life Technologies, Carlsbad, CA, USA) was also applied to parental MDA-MB-231 (231-P) cells and the metastatic subline of MDA-MB-231 (231-B) cells derived from the bones of our animal models, both of which had stable luciferase expression. All these cells were maintained in the incubator without gas exchange with outside air.

Five-week-old female Balb/c nude mice were purchased from Vital River Laboratories (Beijing, China) and bred in the SPF Animal Center of Cancer Institute and Hospital, Chinese Academy of Medical Sciences. All the animal experiments were approved by the Institutional Review Board of the Chinese Academy of Medical Sciences Cancer Institute.

Generation of lentivirus expression vehicles. To facilitate in vivo observation and the following the primary culture of 231-B cells, we constructed a pLVX-IRES-Neo vector containing luciferase gene and then produced the lentivirus by transfecting $6.5 \mu \mathrm{g}$ pLVX-luciferase-Neo vector and corresponding amounts of package vectors into 293T cells, which were seeded in $10-\mathrm{cm}$ flasks before the day of the transfection, following the standard method of Lipofectamine 2000 (Invitrogen). Forty-eight hours after the transfection, supernatant of these $293 \mathrm{~T}$ cells were harvested, centrifuged $\left(3,000 \mathrm{rpm}, 4^{\circ} \mathrm{C}\right)$, filtered using $0.45-\mu \mathrm{m}$ filter flasks, aliquoted and stored at $-80^{\circ} \mathrm{C}(14)$.

For transduction, $10 \mu 1$ supernatant containing lentivirus was added into the MDA-MB-231 cells, which were maintained in $2 \mathrm{ml}$ of L-15 complete culture medium. In addition, $8 \mu \mathrm{g} / \mathrm{ml}$ of polybrene (Sigma-Aldrich) was also present in order to aid transduction. Twenty-four hours later, the medium were replaced by fresh L15 complemented with 10\% FBS. Simultaneously, $400 \mu \mathrm{g} / \mathrm{ml} \mathrm{G} 418$ was used to screen out the uninfected cells.

Establishment of bone metastasis model. The bone metastasis model of breast cancer was described by Yin and colleagues (15). In general, amounts of 231-P cells were harvested using $0.25 \%$ trypsin and $0.53 \mathrm{mM}$ EDTA, resuspended in sterilized PBS, and adjusted to a concentration of $5 \times 10^{6} / \mathrm{ml}$. Then, mice were anesthetized by intraperitoneal injection of $1 \%(\mathrm{w} / \mathrm{v})$ pentobarbital sodium $(90 \mathrm{mg} / \mathrm{kg})$. After confirming that mice were under proper anesthesia, we injected $100 \mu \mathrm{l}$ of the suspended cells into the left ventricle via the third intercostal space with a 29 -gauge needle. A successful injection was confirmed by the pumping of arterial blood into the syringe (16). Mice were sterilized with $70 \%$ alcohol, and then were bred in the following weeks.

In vivo detection of bone metastasis. Mice anesthetized by $1 \%$ (w/v) pentobarbital sodium $(90 \mathrm{mg} / \mathrm{kg}$ ) were intraperitoneally injected with $150 \mathrm{mg} / \mathrm{kg}$ of D-Luciferin (Perkin-Elmer) in DPBS. Bioluminescence images were acquired between 10 and 15 min after injection using Xenogen Corporation Optical in vivo imaging (IVIS Lumina). Image acquisition time at the beginning was $60 \mathrm{sec}$ and then it was reduced in accordance with signal strength to avoid saturation. All images were analyzed with Living Image software. Intensity of bioluminescence was calculated as photons $/ \mathrm{sec} / \mathrm{cm}^{2} / \mathrm{steradian}$ of a region of interest (ROI). Average background reads were obtained from sites of the same mice without bioluminescence.

Moreover, radiographic analysis was adopted to further confirm the bone metastases of 231-P cells. Mice were anesthetized using $1 \%(\mathrm{w} / \mathrm{v})$ pentobarbital sodium $(90 \mathrm{mg} / \mathrm{kg})$ and arranged in proper position and exposed to $\mu \mathrm{CT}$ at $60 \mathrm{kV}$ for 800 msec using an Inveon MM Gantry-LG CT.

Primary cell culture of metastatic breast tumor cells. To isolate tumor cells from the osteolytic lesions, mice with overt bone metastases were sacrificed, and then the affected hindlimbs were separated from the body at the joints. Both ends of the long bones were cut open after skin and muscle was removed using a scalpel. Mouse bone marrow cells as well as tumor cells were washed out by PBS using a 1-ml syringe with a 29G needle. All those cells were collected by centrifuging and washed with PBS before being cultured in $5-\mathrm{cm}$ plates using L-15 medium supplemented with 10\% FBS.

Mouse bone marrow cells that failed to attach to plates and thus could be washed off with PBS after the other cells (almost all of them are tumor cells) adhered to culture plates. After continuous culture using $400 \mu \mathrm{g} / \mathrm{ml} \mathrm{G} 418$ for more than 1 week, we obtained the subline of bone metastasis named as 231-B cells, which were verified by measuring the luciferase activity (Promega).

$R N A$ extraction and expression profiles of miRNA and $m R N A$. Cells with about $80 \%$ confluence on 6-well plates were washed twice using ice-cold PBS, and $1 \mathrm{ml}$ TRIzol (Invitrogen) was added to a well to obtain RNA. Cell lysate in TRIzol was stored at $-80^{\circ} \mathrm{C}$ and then sent for screening differentially expressed miRNA and mRNA using Affymetrix GeneChip miRNA 3.0 Arrays and Human mRNA 4x180K (Agilent Technologies). The quantity and quality of extracted RNA were analyzed by CapitalBio. All data were normalized and further analyses were carried out by the College of Bioinformatics Science and Technology, Harbin Medical University.

Reverse transcription of RNA and real-time PCR. Total RNA was obtained following the standard method of TRIzol extraction. cDNA was synthesized using Quant cDNA with random primers (Tiangen). Moreover, miRNAs were reverse-tran- 
A

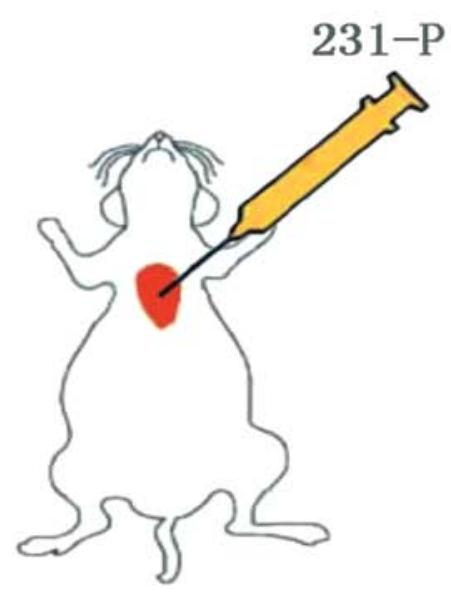

B

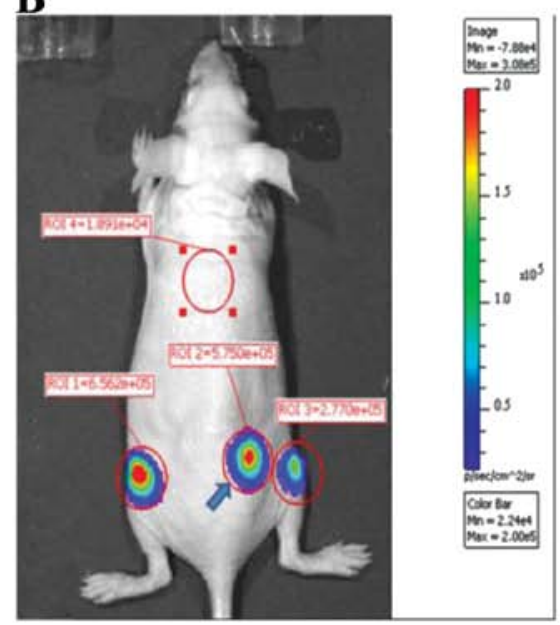

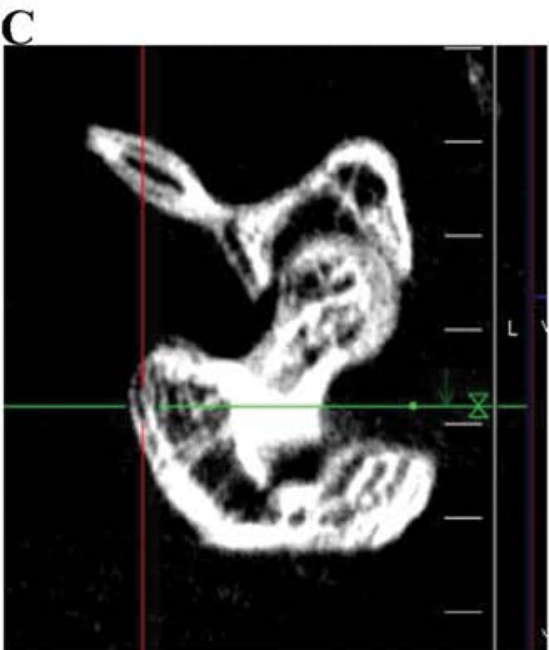

Figure 1. Establishment of the bone metastasis model. (A) Injecting 5x10 $231-\mathrm{P}$ cells into the left ventricle. (B) Twelve weeks later, 3 bone metastasis were detected including distal end of left femur, distal end of right femur and the proximal end of the right femur. The intensity of bioluminescence in bone metastasis was much higher than that of negative background (>10 times). (C) The osteolytic destruction of the proximal end of the right femur which corresponds to bioluminescence signals (arrow in B).

scribed following the instructions with minor alteration (17). In general, one specific reverse primer targeting individual miRNA was designed to complete the reverse transcription. Then, one miRNA-specific forward primer and one universal reverse primer were used in the subsequent real-time PCR detection. Real-time PCR was completed with SYBR Premix Ex Taq ${ }^{\mathrm{TM}}$ II (Takara). The PCR procedure followed the instructions of Takara in StepOne Plus Real-time PCR system (Applied Biosystems). The results were analyzed using the $2^{-\Delta \Delta C t}$ method (18).

Primers used in this study were the following: miR-429 forward, 5'-ACACTCCAGCTGGGTAATACTGTCTGG TAA-3' and miR-429 reverse, 5'-CTCAACTGGTGTCGTGG AGTCGGCAATTCAGTTGAGACGGTTTT-3', universal reverse, 5'-TGGTGTCGTGGAGTCG-3'; U6 forward, CTCGCTTCGGCAGCACA and U6 reverse, AACGCTTCAC GAATTTGCGT.

Transfection of microRNA mimics. HiPerFect agent (Qiagen) was used to deliver miRNA mimics into cells maintained in 6-well plates. According to the manufacturer's instruction, cells were grown to $\sim 70 \%$ confluence and $20 \mathrm{nM}$ miRNA mimics and $12 \mu \mathrm{l}$ of transfection agent was mixed thoroughly in $100 \mu \mathrm{l}$ of Opti-MEM. After $10 \mathrm{~min}$ at room temperature, the mixture was added into cells. The subsequent experiments were performed $48 \mathrm{~h}$ after the transfection.

Cell invasion assays. The upper chambers of Transwells with $8-\mu \mathrm{m}$ membrane pores (Corning) were pre-coated with $60 \mu \mathrm{l}$ Matrigel matrix gel (BD Biosciences) at least $1 \mathrm{~h}$ before seeding of the tested cells. A total of $3 \times 10^{4} 231-\mathrm{B}$ cells in $100 \mu \mathrm{l}$ of L15 medium without FBS were added into the upper chambers and $600 \mu \mathrm{l}$ of L15 medium with 10\% FBS was placed to lower chambers as chemoattractant. Twelve hours later, the upper chambers were removed from lower chambers and then wiped using cotton swabs. The invaded cells were fixed using methanol at room temperature for 15 min, visualized and quantified using crystal violet. Three fields of each chamber were photographed (x10 magnification) and the results were from duplicate chambers and are presented as mean \pm SD. This experiment was independently repeated at least twice.

Immunoblot assay. Cells reaching 80\% confluence on 6-well plates were washed twice using ice-cold PBS and 80-100 $\mu \mathrm{l}$ cell lysis buffer (50 mM Tris- $\mathrm{HCl}, 150 \mathrm{mM} \mathrm{NaCl}, 1 \mathrm{mM}$ EDTA, $1 \%$ Triton-100) with protease inhibitor cocktail (Complete Mini EDTA-free; Roche) added into plates. On ice, cells were carefully collected with scrapers and were subjected to lysis for $30 \mathrm{~min}$. Proper amount of Laemmli buffer was added into 20-30 $\mu \mathrm{g}$ cell lysate and boiled at $95^{\circ} \mathrm{C}$ for $5 \mathrm{~min}$. Then the denaturalized protein was resolved on a $10 \%$ gel. After being transferred to PVDF membrane, the protein blot was detected using corresponding antibodies. The immunoblot images were acquired and analyzed using ImageQuant LAS 4000 System. The primary antibodies used in this experiment were ZEB1 and $\beta$-actin.

Statistical analyses. GraphPad 6 was used to evaluate the statistical significance of data. Unless otherwise indicated, $\mathrm{t}$-test was adopted to calculate the statistical significance. A $\mathrm{P}$-value $<0.05$ was considered significant.

\section{Results}

Breast cancer cells invade bones of Balb/c nude mice. In the present study, we have established a mouse model of bone metastasis. The human mammary carcinoma MDA-MB-231 cells, which constitutively expressed luciferase, were inoculated into the left cardiac ventricle of immunodeficient mice (Fig. 1A). Eight to twelve weeks later, 5 mice eventually developed bone metastasis among the 15 mice under experiment. The bone lesions mainly occur on the long bones of the hind limbs and the ribs, which were observed by Xenogen Corporation Optical in vivo imaging and $\mu \mathrm{CT}$ (Fig. 1B and $\mathrm{C}$ ). 


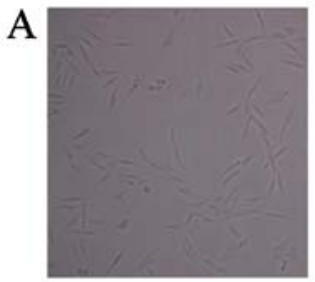

231-P
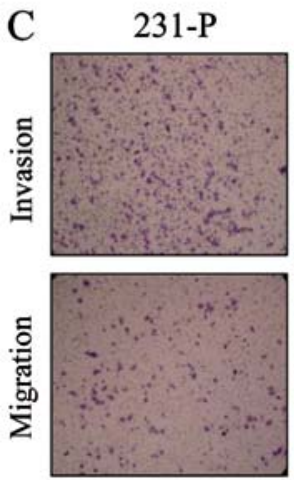

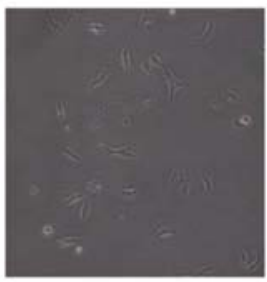

231-B

231-B
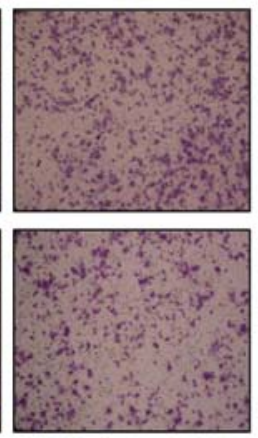

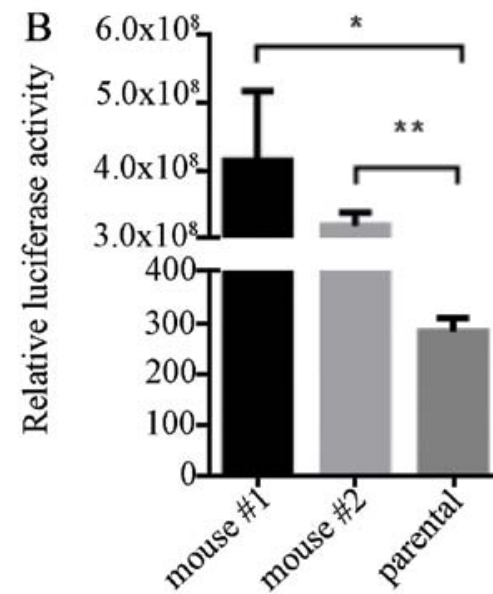

Figure 2. Verification of bone metastatic MDA-MB-231 cells. (A) The distinct morphological characteristics of 231-P cells (left) and 231-B cells (right). (B) MDA-MB-231 cells from bone metastatic lesions of two Balb/c nude mice manifest much stronger luciferase activity than that of cells from the negative control group. (C) In vitro Transwell assays show significantly increased migration and invasion of 231-B cells than that of 231-P cells.

A
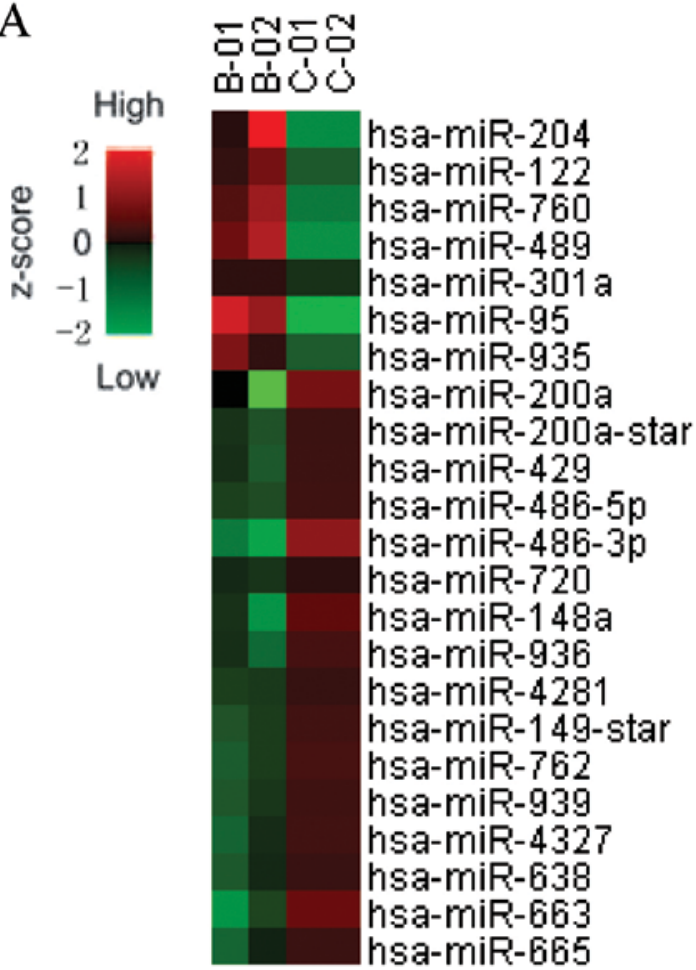
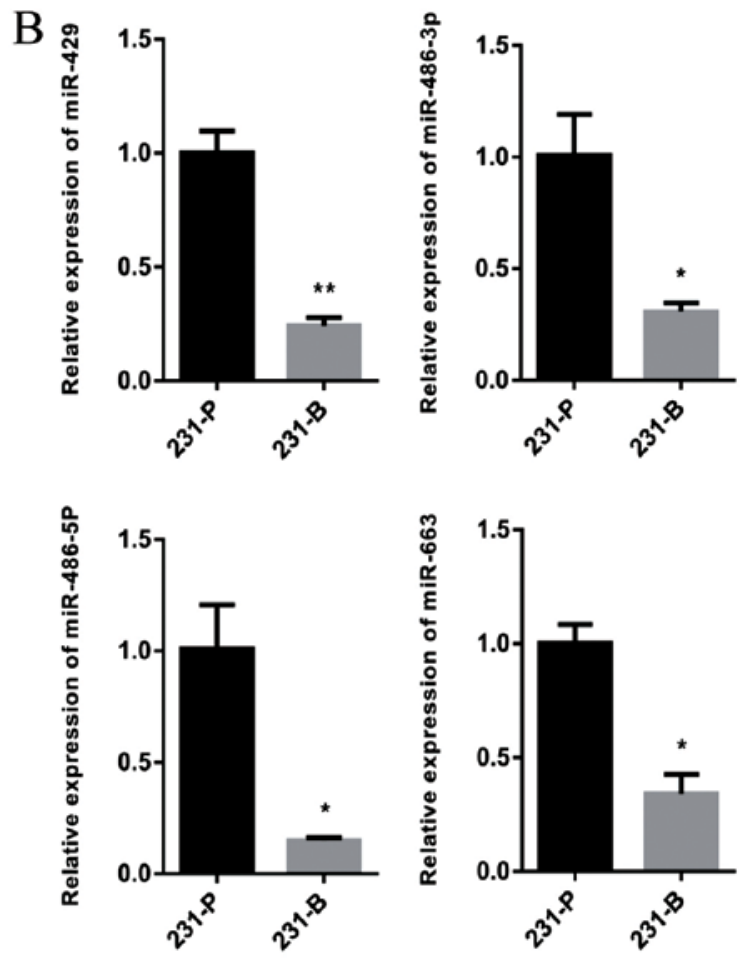

Figure 3. (A) microRNA array shows some of the differently expressed miRNAs. (B) Verifications of microRNA array results by real-time PCR. miR-429, miR-663, miR-486-5p and miR-486-3p showed lower expression in 231-B cells than that in 231-P cells.

Primary culture of bone metastatic breast cancer cells. After verifying the presence of bone metastases, we collected the metastatic tumor cells from the affected bones. G418 were added into the culture medium to wipe out non-malignant cells. After about 10 days, we obtained 231-B cells (Fig. 2A) of which morphology was different from 231-P cells. Then, we tested the luciferase activity of 231-B cells, obtaining much higher luciferase activity compared with the negative control cells (Fig. 2B). Moreover, in vitro invasion assay showed that 231-B cells had stronger invading capability than 231-P cells (Fig. 2C). Thus, we obtained bone metastatic breast tumor cells from the established model. 

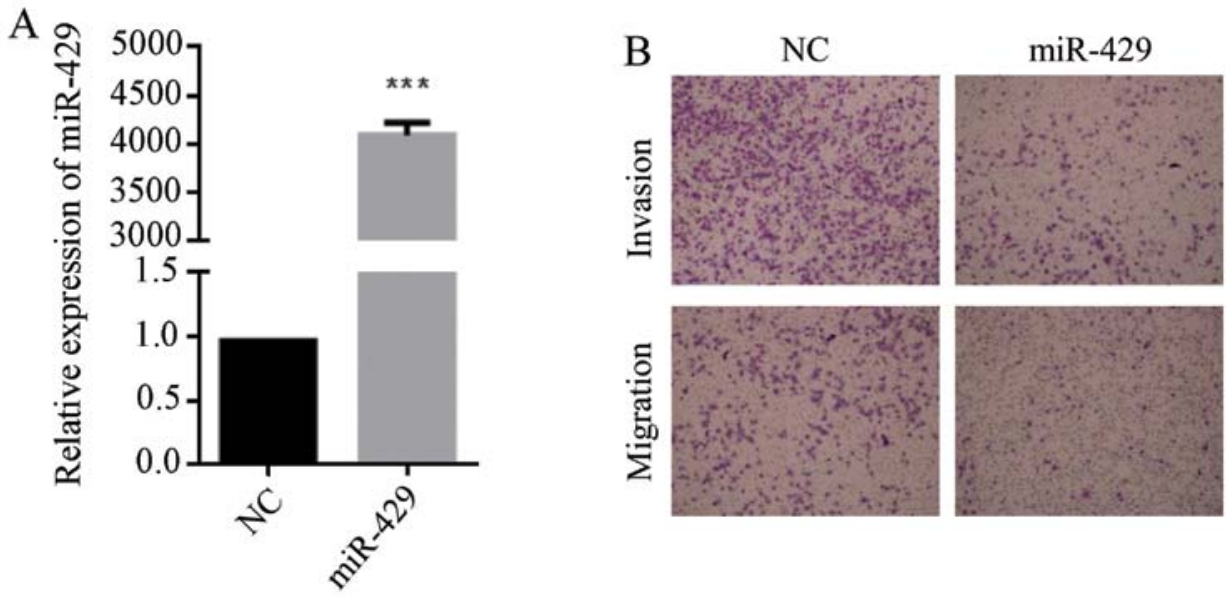

Figure 4. miR-429 inhibits migration and invasion of MDA-MB-231 cells in vitro. (A) Overexpression of miR-429 in 231-B cells, showing the efficiency of transfection. (B) Increased miR-429 significantly hampers the motility and invasiveness of 231-B cells in vitro.

Table I. The candidate targets of miR-429, which were verified to play a vital role in the development of certain cancer, were predicted in six algorithms.

\begin{tabular}{|c|c|c|c|c|c|c|}
\hline & TargetScan & DIANA-microT & microRNA.org & $\operatorname{miRDB}$ & RNA22-HSA & PITA \\
\hline ZEB1 & + & + & + & + & + & + \\
\hline ZEB2 & + & + & + & + & + & \\
\hline WIPF1 & + & + & + & + & & + \\
\hline MARCKS & + & + & + & + & & + \\
\hline TRIM33 & + & + & + & + & & + \\
\hline WASF3 & + & + & + & + & & + \\
\hline CRKL & + & + & + & + & & + \\
\hline WAPAL & + & + & + & + & & + \\
\hline NTRK2 & + & & + & + & + & + \\
\hline
\end{tabular}

Differentially expressed miRNAs of 231-P and 231-B cells. RNA samples from 231-P and 231-B cells were prepared and analyzed using Affymetrix GeneChip miRNA 3.0 Arrays. Among the detected miRNAs that amounted to 3,949, 118 miRNAs manifested differential expression between 231-P and 231-B cells, of which 16 upregulated whereas 102 downregulated $>2$-fold $(\mathrm{P}<0.05)$. Some of the differentially expressed miRNA are shown in Fig. 3A. Subsequent GO enrichment analysis showed that the functions of these miRNAs focused on 'regulation of cell death' $\left(\mathrm{P}=1.49 \mathrm{xe}^{-6}\right)$, 'signaling' $\left(\mathrm{P}=3.25 \mathrm{xe}^{-6}\right)$, 'regulation of localization' $(\mathrm{P}=6.31$ $\left.\mathrm{xe}^{-6}\right)$ and 'regulation of cell migration' ( $\left.\mathrm{P}=0.00028\right)$.

In addition, miR-429, miR-663, miR-486-5p and miR486-3p were verified in RNA samples derived from 231-P and 231-B cells by real-time PCR assay. Results showed that these miRNAs indeed had distinct expression patterns, indicating that they probably play important roles in bone metastasis of breast cancer cells (Fig. 3B).

miR-429 suppresses invasion of 231-B cells in vitro. Previous reports showed that miR-429 could inhibit migration and metastasis of several types of cancers. Overexpression of miR-429 inhibits invasion and promotes apoptosis in esopha- geal carcinoma cells by targeting Bcl-2 and SP1 (19). miR-429 inhibits cells invasion by targeting Onecut 2 in colorectal carcinoma (20). Our results were in line with the above investigations.

We noted that miR-429 expression was significantly lower in 231-B cells than that of 231-P cells (Fig. 3B). We transfected miR-429 mimics into 231-B cells, which was verified by realtime PCR assay (Fig. 4A), and found that overexpression of miR-429 could dramatically reduce the migration and invasiveness of 231-B cells (Fig. 4B). Taken together, miR-429 probably regulated bone metastasis of breast cancer cells in a negative manner.

miR-429 mediates inhibitory function by targeting multiple genes. To gain insight into the roles of miR-429 inhibiting bone metastasis of MDA-MB-231 cells, several computational algorithms (including TargetScan, DIANA-microT, microRNA.org, miRDB, RNA22-HSA and PITA) were used to predict the candidate targets of miR-429, ZEB1, ZEB2, WIPF1, MARCKS, TRIM33, WASF3, CRKL, WAPAL and NTRK2 were predicted as candidate targets of miR-429 (Table I). Fig. 5A shows the possible regulation network between miRNAs and mRNAs. Then, we combined the 

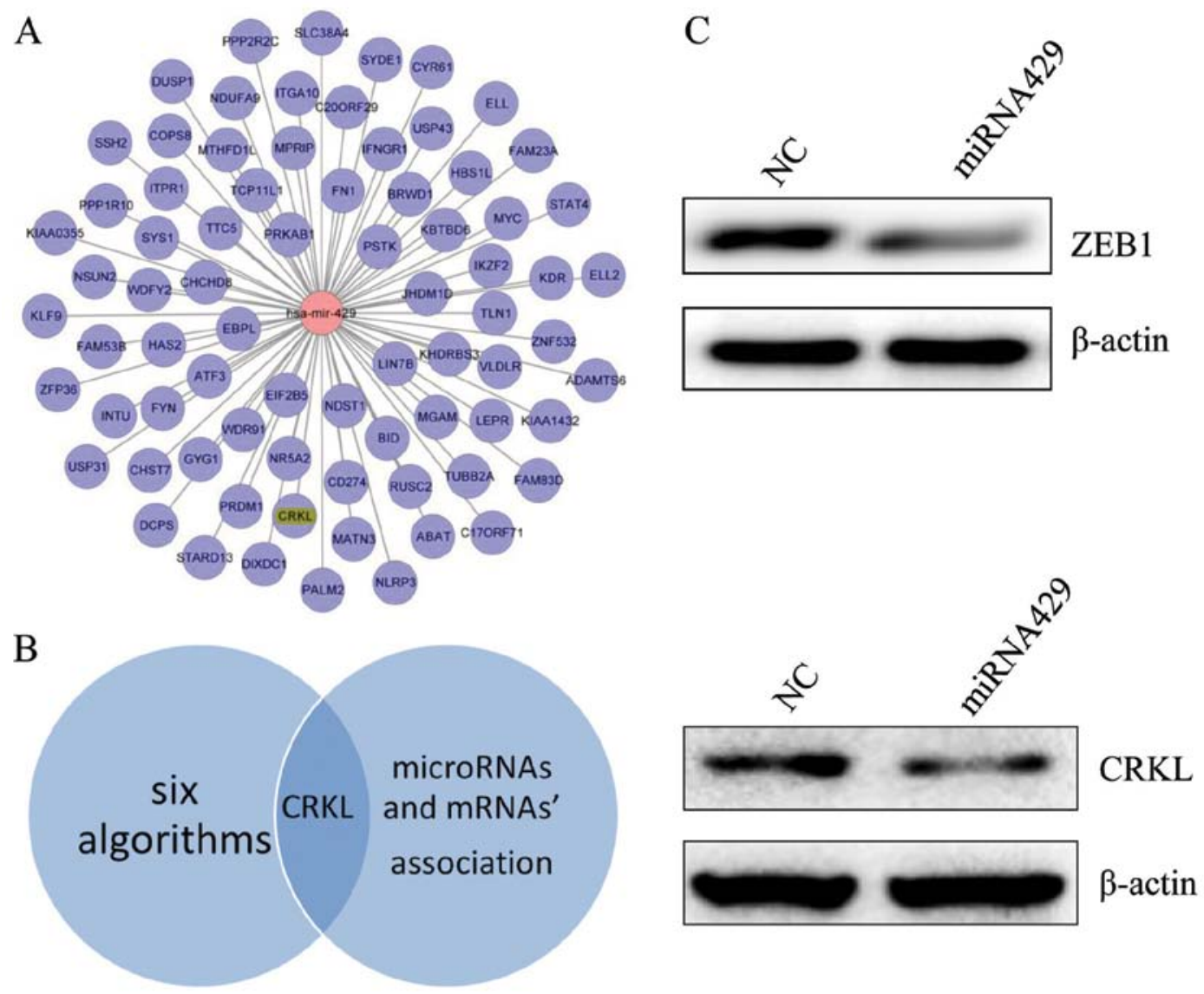

Figure 5. Search for the functional targets of miR-429. (A) The microRNAs and their potential targets detected in a global expression assay. (B) The map illustrates that CRKL is probably a new genuine target of miR-429 in bone metastasis of breast cancer cells through cross-evaluating results from in silico search and expression array. (C) Immunoblot assay shows that overexpression of miR-429 in 231-B cells results in reduced expression of ZEB1 and CRKL.

in silico analysis of miR-429 targets and global transcriptional profile (Fig. 5B). Among all these results, CRKL was the target gene on both sides, indicating this gene was probably a fundamental node in controlling bone metastasis of breast cancer. Moreover, immunoblot assay showed that miR-429 reduced ZEB1 and CRKL significantly, which is a classical EMT inducer in breast cancer (Fig. 5C). Collectively, miR-429 negatively modulated several key invasion and metastasis inducers to suppress motility of breast cancer cells.

\section{Discussion}

Breast cancer morbidity ranks the first accounting for $29 \%$ in all the women suffering cancer, and the mortality ranks the second accounting for $14 \%$ among the women cancer victims in western countries $(21,22)$. Although great progress has been made toward preventing and treating breast cancer with surgical operation, chemotherapy, and radiation therapy, metastasis of breast cancer still remains one grave clinical challenge due to the limits of early detection and effective treatment. Breast cancer is likely to form distant metastases in lung, bone and liver (23). A myriad of factors influence malignant mammary cells to colonize in bones $(24,25)$. In this investigation, we set up an in vivo model to study how microRNAs regulate bone metastases of breast cancer cells.

We established a bone metastasis model using MDA-MB231 cells, and further miRNA array analysis revealed the altered expression of more than 100 miRNAs between 231-P and 231-B cells. Among these miRNAs, several have already been found to get involved in invasion and metastasis of tumors. miR-663 could inhibit proliferation and invasion of glioblastoma in vitro and in vivo by directly targeting PIK3CD, thus, reducing the expression of three downstream effectors: CCND1, MMP2 and MMP7 (26). On the other hand, in castration-resistant prostate cancer cells, miR-663 acted as OncomiR, which could promote invasion of malignant cells. Moreover, this miRNA correlated with TNM stage and could be used as an independent prognostic marker in clinical recurrence (27). Besides, miR-486-5p, expression of which in 231-B cells was decreased by $70 \%$ compared with that of 231-P cells, was recently reported to inhibit metastasis of lung cancer by reducing the protein level of ARHGAP5 (28). Taken together, most array results from comparison between 231-P and 231-B cells were in line with previous literature and these differentially expressed miRNAs are probably implicated in bone metastasis of breast cancer.

miR-429 is a member of the miR-200 family, four members of this family have been found to play important roles in the regulation of EMT in various types of tumors (29). EMT is widely accepted as an essential mechanism to prompt malignant cells to invade and colonize distant organs (30). In the present study, we showed that downregulation of miR-429 could promote bone metastasis of breast cancer, which is in line with previously reported results in several types of 
cancers. Besides in breast cancer, overexpression of miR-429 suppressed invasion and metastasis of colorectal carcinoma and ovarian cancer cells, respectively $(20,31)$.

miRNAs usually function through base-pairing specific sequences located in 3'-UTRs of targeted genes to hinder translation of mRNA or enhance degradation of mRNA (32). ZEB1 is the most well studied target of miR-429, since this transcription factor is an important EMT inducer (33). Besides ZEB1, several candidate targets of miR-429 were also identified essential for invasion and metastasis in cancer models. For example, Onecut 2 was proved to be one bona fide target of miR-429 in colon cancer (20). Combined analysis of expression array results and in silico target search revealed CRKL as a common candidate. CRKL protein is a kinase harboring $\mathrm{SH} 2$ and $\mathrm{SH} 3$ domains. CRKL transformed fibroblasts and received signals from BCR-ABL tyrosine kinase (34-36). CRKL influences integrin mediated adhesion to fibronectin (37). For cellular motility, CRKL enhanced motion through forming a complex with $\mathrm{Cbl}$ and $\mathrm{C} 3 \mathrm{G}$ to relay signals in hematopoietic $\mathrm{Ba} / \mathrm{F} 3$ cells (38). In addition to promoting invasion of cells, CRKL also could suppress caspase- 8 mediated apoptosis, which was also essential for distant metastasis (39). Another research group also found the involvement of CRKL in integrin-triggered cell migration, and they further identified that CRKL performed by acting as downstream effector of Src (40). Considering that CRKL is important in proliferation, migration and evading apoptosis, we speculate that CRKL is probably essential in bone metastasis of breast cancer, however this needs further investigation.

In conclusion, we established an in vivo bone metastasis model of breast cancer, and we obtained a wealth of information on how mammary malignant cells invade and finally form overt metastases in bones. Further research is urgently needed to clarify the complicated mechanisms underlying this fatal pathological condition to provide valuable prevention and treatment targets.

\section{Acknowledgements}

We appreciate our colleagues in the laboratory of Dr Zhihua Liu for helpful discussion, and Professor Burton Yang for his critical reading of this manuscript.

\section{References}

1. Yang W, Lam P, Kitching R, et al: Breast cancer metastasis in a human bone NOD/SCID mouse model. Cancer Biol Ther 6: 1289-1294, 2007.

2. Xia TS, Wang GZ, Ding Q, et al: Bone metastasis in a novel breast cancer mouse model containing human breast and human bone. Breast Cancer Res Treat 132: 471-486, 2012.

3. Fidler IJ: Critical determinants of metastasis. Semin Cancer Biol 12: 89-96, 2002.

4. Li Z, Schem C, Shi YH, Medina D and Zhang M: Increased COX2 expression enhances tumor-induced osteoclastic lesions in breast cancer bone metastasis. Clin Exp Metastasis 25: 389-400, 2008.

5. Rosol TJ, Tannehill-Gregg SH, Corn S, Schneider A and McCauley LK: Animal models of bone metastasis. Cancer Treat Res 118: 47-81, 2004

6. Lynam-Lennon N, Maher SG and Reynolds JV: The roles of microRNA in cancer and apoptosis. Biol Rev Camb Philos Soc 84: 55-71, 2009.
7. Qiao J, Lee S, Paul P, et al: miR-335 and miR-363 regulation of neuroblastoma tumorigenesis and metastasis. Surgery 154: 226-233, 2013.

8. Yi C, Wang Q, Wang L, et al: MiR-663, a microRNA targeting $\mathrm{p} 21^{\mathrm{WAF} 1 / \mathrm{CIP1}}$, promotes the proliferation and tumorigenesis of nasopharyngeal carcinoma. Oncogene 31: 4421-4433, 2012.

9. Pan J, Hu H, Zhou Z, et al: Tumor-suppressive miR-663 gene induces mitotic catastrophe growth arrest in human gastric cancer cells. Oncol Rep 24: 105-112, 2010.

10. Yau WL, Lam CS, Ng L, et al: Over-expression of miR-106b promotes cell migration and metastasis in hepatocellular carcinoma by activating epithelial-mesenchymal transition process. PLoS One 8: e57882, 2013.

11. Dynoodt P, Speeckaert R, De Wever O, et al: miR-145 overexpression suppresses the migration and invasion of metastatic melanoma cells. Int J Oncol 42: 1443-1451, 2013.

12. Li LZ, Zhang CZ, Liu LL, et al: miR-720 inhibits tumor invasion and migration in breast cancer by targeting TWIST1. Carcinogenesis 35: 469-478, 2014.

13. Wang C, Bian Z, Wei D and Zhang JG: miR-29b regulates migration of human breast cancer cells. Mol Cell Biochem 352: 197-207, 2011.

14. Kutner RH, Zhang XY and Reiser J: Production, concentration and titration of pseudotyped HIV-1-based lentiviral vectors. Nat Protoc 4: 495-505, 2009.

15. Yin JJ, Selander K, Chirgwin JM, et al: TGF-beta signaling blockade inhibits PTHrP secretion by breast cancer cells and bone metastases development. J Clin Invest 103: 197-206, 1999.

16. Kang Y, Siegel PM, Shu W, et al: A multigenic program mediating breast cancer metastasis to bone. Cancer Cell 3: 537-549, 2003

17. Chen C, Ridzon DA, Broomer AJ, et al: Real-time quantification of microRNAs by stem-loop RT-PCR. Nucleic Acids Res 33: e179, 2005

18. Schmittgen TD and Livak KJ: Analyzing real-time PCR data by the comparative C(T) method. Nat Protoc 3: 1101-1108, 2008.

19. Wang Y, Li M, Zang W, et al: MiR-429 up-regulation induces apoptosis and suppresses invasion by targeting Bcl-2 and SP-1 in esophageal carcinoma. Cell Oncol (Dordr) 36: 385-394, 2013.

20. Sun Y, Shen S, Liu X, et al: miR-429 inhibits cells growth and invasion and regulates EMT-related marker genes by targeting Onecut 2 in colorectal carcinoma. Mol Cell Biochem 390: 19-30, 2014.

21. Smith RA, Brooks D, Cokkinides V, Saslow D and Brawley OW: Cancer screening in the United States, 2013: a review of current American Cancer Society guidelines, current issues in cancer screening, and new guidance on cervical cancer screening and lung cancer screening. CA Cancer J Clin 63: 88-105, 2013.

22. Siegel R, Naishadham D and Jemal A: Cancer statistics, 2013. CA Cancer J Clin 63: 11-30, 2013.

23. Weigelt B, Peterse JL and van't Veer LJ.: Breast cancer metastasis: markers and models. Nat Rev Cancer 5: 591-602, 2005.

24. Mundy GR: Metastasis to bone: causes, consequences and therapeutic opportunities. Nat Rev Cancer 2: 584-593, 2002.

25. Weilbaecher KN, Guise TA and McCauley LK: Cancer to bone: a fatal attraction. Nat Rev Cancer 11: 411-425, 2011.

26. Shi Y, Chen C, Zhang X, et al: Primate-specific miR-663 functions as a tumor suppressor by targeting PIK3CD and predicts the prognosis of human glioblastoma. Clin Cancer Res 20: 1803-1813, 2014.

27. Jiao L, Deng Z, Xu C, et al: miR-663 induces castration-resistant prostate cancer transformation and predicts clinical recurrence. $\mathrm{J}$ Cell Physiol 229: 834-844, 2014.

28. Wang J, Tian X, Han R, et al: Downregulation of miR-486-5p contributes to tumor progression and metastasis by targeting protumorigenic ARHGAP5 in lung cancer. Oncogene 33: 1181-1189, 2014.

29. Korpal M and Kang Y: The emerging role of miR-200 family of microRNAs in epithelial-mesenchymal transition and cancer metastasis. RNA Biol 5: 115-119, 2008.

30. Polyak K and Weinberg RA: Transitions between epithelial and mesenchymal states: acquisition of malignant and stem cell traits. Nat Rev Cancer 9: 265-273, 2009.

31. Chen J, Wang L, Matyunina LV, Hill CG and McDonald JF: Overexpression of miR-429 induces mesenchymal-to-epithelial transition (MET) in metastatic ovarian cancer cells. Gynecol Oncol 121: 200-205, 2011.

32. Pasquinelli AE: MicroRNAs and their targets: recognition, regulation and an emerging reciprocal relationship. Nat Rev Genet 13: 271-282, 2012. 
33. Peinado H, Olmeda D and Cano A: Snail, Zeb and bHLH factors in tumour progression: an alliance against the epithelial phenotype? Nat Rev Cancer 7: 415-428, 2007.

34. Ten HJ, Kaartinen V, Fioretos T, et al: Cellular interactions of CRKL, and SH2-SH3 adaptor protein. Cancer Res 54: 2563-2567, 1994.

35. Heaney C, Kolibaba K, Bhat A, et al: Direct binding of CRKL to $\mathrm{BCR}-\mathrm{ABL}$ is not required for BCR-ABL transformation. Blood 89: 297-306, 1997.

36. Senechal K, Halpern J and Sawyers CL: The CRKL adaptor protein transforms fibroblasts and functions in transformation by the BCR-ABL oncogene. J Biol Chem 271: 23255-23261, 1996.
37. Uemura N, Salgia R, Ewaniuk DS, Little MT and Griffin JD: Involvement of the adapter protein CRKL in integrin-mediated adhesion. Oncogene 18: 3343-3353, 1999.

38. Uemura $\mathrm{N}$ and Griffin JD: The adapter protein Crkl links $\mathrm{Cbl}$ to C3G after integrin ligation and enhances cell migration. J Biol Chem 274: 37525-37532, 1999.

39. Graf R, Barbero S, Keller N, et al: Src-inducible association of CrkL with procaspase- 8 promotes cell migration. Cell Adh Migr 7: 362-369, 2013.

40. Li L, Guris DL, Okura M and Imamoto A: Translocation of CrkL to focal adhesions mediates integrin-induced migration downstream of Src family kinases. Mol Cell Biol 23: 2883$2892,2003$. 